\title{
Impedance model of metal-dielectric nanocomposites produced by ion-beam sputtering in vacuum conditions and its experimental verification for thin films of $(\mathrm{FeCoZr})_{x}(\mathrm{PZT})_{(100-x)}$
}

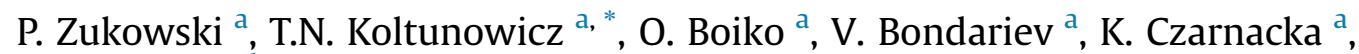 \\ J.A. Fedotova ${ }^{\text {b }}$, A.K. Fedotov ${ }^{\text {c }}$, I.A. Svito ${ }^{c}$ \\ a Lublin University of Technology, 20-618 Lublin, Poland \\ b National Centre for Particles and High Energy Physics of BSU, 220040 Minsk, Belarus

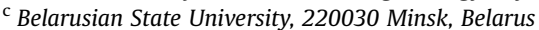

\section{A R T I C L E I N F O}

\section{Article history:}

Received 27 September 2014

Received in revised form 12 April 2015

Accepted 25 April 2015

Available online $\mathrm{xxx}$

\section{Keywords:}

AC measurement

Electron transport

Nanocomposites metal-dielectric

\begin{abstract}
A B S T R A C T
The paper presents results of testing electric properties (resistance, capacity and phase angle in an equivalent parallel circuit) of ferromagnetic alloy-dielectric nanocomposites $(\mathrm{FeCoZr})_{x}(\mathrm{PZT})_{(100-x)}$ produced by ion-beam sputtering in vacuum conditions. The measurements have been performed using alternating current within the frequency range of $50 \mathrm{~Hz}-1 \mathrm{MHz}$ for measuring temperatures ranging from $77 \mathrm{~K}$ to $373 \mathrm{~K}$. In nanocomposites $\left(\mathrm{CoFeZr}_{x}(\mathrm{PZT})_{(100-x)}\right.$, produced by ion beam sputtering using a beam of combined argon and oxygen ions, for $x$ approaching the percolation threshold, frequency dependences of the phase angle $\varphi$ that resemble those occurring in RLC parallel circuits have been observed. In the low frequency area, the phase angle of $90^{\circ} \leq \varphi<0^{\circ}$ occurs. It corresponds to the capacitive type of conduction. In the high frequency area, the inductive type of conduction with $0^{\circ} \leq \varphi \leq 90^{\circ}$ occurs. At the resonance frequency $f_{0}$, characterized by the phase angle of $\varphi=0^{\circ}$, the capacity value reaches its strong local minimum. A theoretical basis for a model of the AC hopping conduction for metal-dielectric nanocomposites has been developed and on that basis frequency dependences of the phase angle, resistance and capacitive current density components have been analyzed. The obtained theoretical and experimental results have been compared.
\end{abstract}

() 2015 Elsevier Ltd. All rights reserved.

\section{Introduction}

Development production and testing of nanomaterials and nanostructures are the most interesting and prospective areas in materials science. The topicality of these research areas follows from the fact that the materials are characterized by versatile structures and phase composition of the nanoscale dimension $\mathrm{A}$ number of their physical-chemical properties, significantly differ from the properties of materials of the micro- or millimetre-scale [1]. Differences in the characteristics of nanocomposites as compared to massive materials result from the surface (surface energy) influence along with the particle transition into the nanosize and, in several cases, from the quantum-dimensional effects [1].

\footnotetext{
* Corresponding author. Fax: +48 815384575.

E-mail address: t.koltunowicz@pollub.pl (T.N. Koltunowicz).
}

From among a wide range of nanomaterials and nanostructures, it is soft-magnetic granular nanomaterials that deserve particular attention. They contain ferromagnetic alloy nanoparticles distributed in a dielectric or ferroelectric matrix [2-6]. Such materials can be used in magneto-electronics, including spintronics, for sensors of electrical, magnetic and non-electric values and for a number of other applications [7-15]. Some of the nanocomposites are characterized by interesting tribological and mechanic properties $[16,17]$ as well as corrosive properties [18].

A standard way to obtain information about electro-physical properties of materials is to use temperature dependences of resistance and of the Hall effect measured with the use of direct current [19-21]. As most of electronic devices, wherein nanocomposites can be applied, operate in AC circuits, our testing of the nanocomposite electrical properties has been performed with the use of AC devices.

Another advantage of the AC-based testing is that it makes it possible to determine many essential electric parameters of 
nanocomposites such as conductivity, permittivity, phase angle and loss tangent as a function of frequency. Additionally, AC measurements of electro-physical properties performed for a wide frequency range make possible to obtain information on the charge transfer mechanism and specifically to distinguish between the band conduction and the hopping conduction. In the case of common conduction in one of allowed energy bands, the conductivity vs. frequency dependence does not occur, whereas for hopping charge transfer, the conductivity increases with rise in measuring frequency [22].

In the case of hopping exchange of electrons (tunneling) between potential wells that are situated at nanometer distances from each other, when an electron hops from one well to another an electrical dipole forms. The well, from which the electron hops, gets positively charged, while the well to which the electron hops, obtains the negative charge. In the case of conductivity produced by electron tunneling between potential wells, the relaxation time $\tau$ is an important parameter. This is the time period from the dipole formation to the return of the hopping electron to the well wherefrom its hop has been initiated. The relaxation time value is of significant impact on both the permittivity and the conductivity resulting from the hopping electron exchange, and on the frequency range, where these phenomena occur.

In Ref. [23] a model of the AC and DC hopping electron transfer has been presented and the real component of current density has been analyzed. It follows from the model that in materials of the hopping conduction mechanism in the area of low angular frequency values $(\omega \ll 1 / \tau)$ conductivity assumes a constant value (DC conductivity). This result conforms with the results of other research works on insulating materials. For the intermediate frequency area $(\omega \sim 1 / \tau)$, the model predicts an increase of conductivity given by the formula:

$\sigma(\omega) \propto \omega^{\alpha}$

where: $\sigma(\omega)$ - conductivity; $\omega=2 \pi f-$ angular frequency; $f$ - frequency; $\alpha$ - frequency coefficient.

The value of the frequency coefficient $\alpha$ depends on the probability $(1-p)$ of the electron return to the well from which it has hopped to another well, while the probability $p$ determines the flow of direct current and the value of its conductivity. This indicator describes by how many times the DC conductivity is lower than the high frequency conductivity. In the high frequency area $(\omega>1 / \tau)$ the conductivity stops increasing and tends to assume a constant value.

The model of the DC and AC hopping electron transfer for the real component has been experimentally verified based on testing the frequency dependence of conductivity for semiconductors that contain potential wells and for nanocomposites [24,25].

For the measurement purposes, nanocomposites of the metaldielectric structure and the composition of $(\mathrm{FeCoZr})_{x}(\mathrm{PZT})_{(100-x)}$ have been selected. The dielectric phase PZT is an acronym for the first letters of the main matrix elements - $\left(\mathrm{Pb}_{81} \mathrm{Sr}_{4}\left(\mathrm{Na}_{50}\right.\right.$ $\left.\left.\mathrm{Bi}_{50}\right)_{15}\left(\mathrm{Zr}_{57.5} \mathrm{Ti}_{42.5}\right)\right) \mathrm{O}_{3}$.

Direct observation of the FeCoZr-based nanoparticles inside the PZT matrix can be very problematic from the experimental viewpoint. This is because both the matrix and the nanoparticles contain heavy elements $(\mathrm{Zr}, \mathrm{Ti})$ and cannot be clearly separated by TEM. However, a combination of techniques (Mössbauer spectroscopy, Raman spectroscopy, XRD, X-ray absorption) can clearly reveal the formation of well-separated $\alpha-\mathrm{FeCo}(\mathrm{Zr})$, FeCo-based oxides and PZT-like phases in films [6,26-28].

$(\mathrm{FeCoZr})_{x}(\mathrm{PZT})_{(100-x)}$ films contain fully oxidized superparamagnetic nanoparticles of either $\mathrm{Fe}(\mathrm{Co})_{3} \mathrm{O}_{4}$ or $\mathrm{Fe}(\mathrm{Co})_{2} \mathrm{O}_{3}$ oxides over the whole range of the $\mathrm{x}$-values studied $(x=35-81$ at.\%) [26-28]. The XRD analysis also proves the absence of a non-oxidized $\alpha$-FeCo(Zr) phase in $(\mathrm{FeCoZr})_{x}(\mathrm{PZT})_{(100-x)}$ films obtained at higher oxygen pressure. Thus, these films consist of $(\mathrm{Fe}, \mathrm{Co}, \mathrm{Zr})_{\mathrm{x}} \mathrm{O}_{\mathrm{y}}$ granules in the PZT-matrix [29].

The AC-based measurements have shown that in the materials $(\mathrm{FeCoZr})_{x}(\mathrm{PZT})_{(100-x)}$, produced by ion sputtering in vacuum conditions and then subjected to annealing in a tubular furnace, a phenomenon of coilless-like inductance occurs. It results in the occurrence of positive phase angles [6,30-32].

The objective of the presented project has been to develop an impedance model of metal-dielectric nanocomposites and to verify it experimentally by comparing the obtained results to the results of AC measurements performed for electric properties of nanocomposites $(\mathrm{FeCoZr})_{x}(\mathrm{PZT})_{(100-x)}$ produced by ion sputtering in vacuum conditions using a complex metal-dielectric target.

\section{Impedance model of metal-dielectric nanocomposites}

The paper presents a model of the DC and AC hopping charge exchange based on the analysis of experimental results, presented in Refs. [33-36]. The model includes the following assumptions:

- the material contains neutral potential wells situated close to each other, where electrons are located (Fig. 1a);

- potential wells are metallic-phase nanoparticles separated by a dielectric matrix;

a)

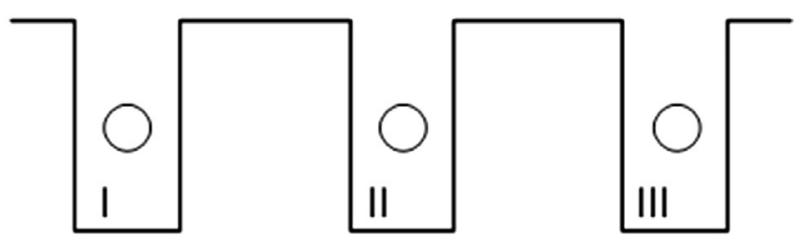

b)

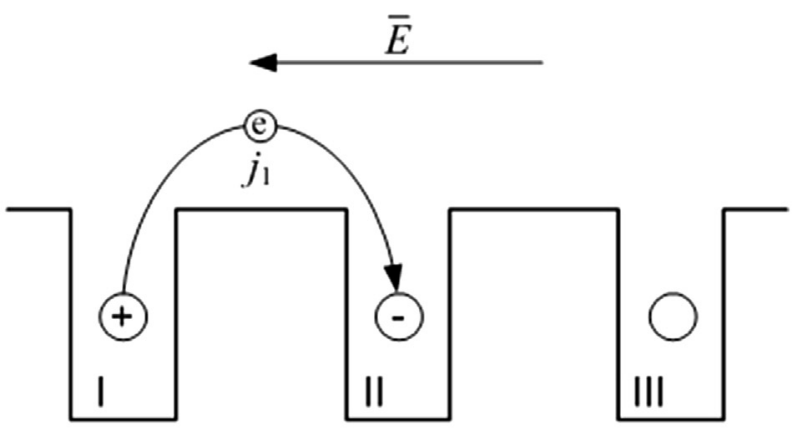

c)

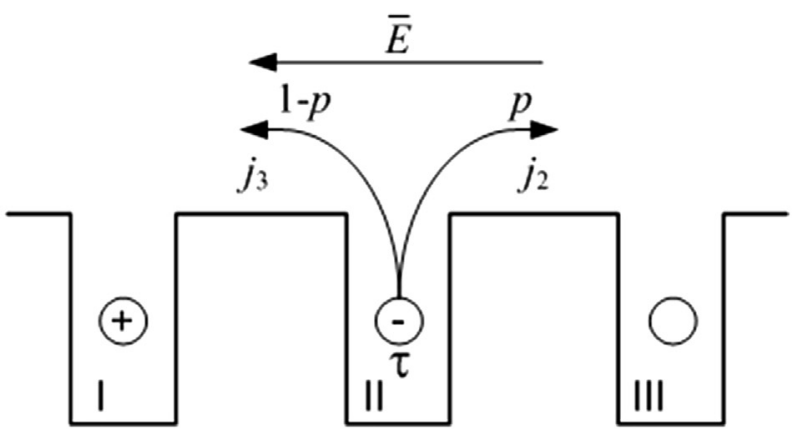

Fig. 1. Potential wells before and after electron jump. 
- the external exciting alternating or constant electric field is weak and does not affect the probability of electron hopping $P(T)$ from one neutral potential well to another; it merely brings about some asymmetry of hops prior to the occurrence of direct current or low-frequency alternating current in-phase with the exciting electric field.

Further analysis uses the below formula for hopping probability as the time unit function $P(T)$ given in Ref. [22]:

$P(T)=P_{0}(T) \exp \left(-\beta \alpha r-\frac{\Delta E}{k T}\right)$

where: $r$ - distance covered by the electron hop, $\exp (-\beta \alpha r)$ - rate of the electron wave function collapse outside the potential well, $\alpha$ - value approaching the inverse localization radius of the hopping electron, $\beta$ - numeric factor, whose value approaches 2 [37],

$\Delta E$ - activation energy of electron hops, $k$ - Boltzman constant, $T$ - temperature, $P_{0}-$ numeric factor.

The value of the above-mentioned weak alternating electric field can be determined as follows:

$E=E_{0} \sin \omega t$

where: $\omega$ - angular frequency, $E_{0}$ - amplitude of the electric field.

Under the influence of that field, a current density $\mathrm{j}_{1}$ is produced (Fig. 1b):

$j_{1}=\sigma \cdot E=\sigma \cdot E_{0} \sin \omega t$.

Once an electron hops from one neutral well to another (Fig. 1b), an electric dipole forms, which leads to an additional thermally activated polarization of the material and an increase of its permittivity [38]. Once an electron has hopped from one neutral well to another it stays there for the time $\tau$ and there are then two options for its further hops. The first option is that the electron hops to the next (third) well in the direction determined by the external electric field (Fig. 1c). The probability of such a hop is denoted by $p$. The current density component produced by such a hop can be described as follows:

$j_{2}=\sigma \cdot E_{0} \cdot p \sin [\omega(t-\tau)]$.

The other option is that, having stayed in the second well for the time $\tau$, the electron hops back to the first well (Fig. 1c). The probability of such a hop is $(1-p)$. As a result, the dipole vanishes and the third current density component occurs. This component can be written as follows:

$j_{3}=-\sigma \cdot E_{0}(1-p) \sin [\omega(t-\tau)]$.

Based on the above considerations, the time $\tau$ can be referred to as the dipole lifetime.

The resultant conduction current density (formulas (4)-(6)) can be described by the formula:

$j_{P}=j_{1}+j_{2}+j_{3}=\sigma E_{0}\{\sin \omega t-(1-2 p) \sin [\omega(t-\tau)]\}$.

It should be noted that equations (4)-(7) are valid only for the measuring temperature area of $T<500 \mathrm{~K}$, when $P(T) \cdot \tau \ll 1$, which means, that only a small part of the potential wells has been charged by the electron hopping.

The components $j_{2}$ and $j_{3}$ are phase shifted relative to $j_{1}$, hence the resultant current density $j_{P}$ includes both the real and imaginary components. It follows from the formula (7) that in materials having hopping conductivity, as opposed to the band conduction case, the conduction current density is phase-shifted relative to the exciting electric field.
It follows from the second Maxwell's equation that in dielectric materials, apart from the component related to the electron transport (conductive current), a polarization-related displacement current also occurs. The density of this current, often referred to as capacitive current, is phase-shifted by $-90^{\circ}$ relative to the exciting field and can be described by the following formula:

$j_{c}=\omega \cdot \varepsilon_{r} \cdot \varepsilon_{0} \cdot \mathrm{E}_{0} \sin \left[\omega \cdot t-\frac{\pi}{2}\right]$,

where: $\varepsilon_{r}$ - relative permittivity, $\varepsilon_{0}$ - vacuum permittivity.

Based on the equations (4)-(8), real and imaginary current density components can be derived, taking into account that $(-\omega \tau)=-\sin (\omega \tau)$ and $\cos (-\omega \tau)=\cos (\omega \tau)$. The current density $j_{1}$ is in-phase with the exciting electric field, consequently it includes only the real component.

Components $j_{2}$ and $j_{3}$ are in the same phase, hence:

$j_{2}+j_{3}=-\sigma \cdot E_{0} \cdot \sin [\omega(t-\tau)] \cdot(1-2 p)$.

The real current density component of the formula (7) can be written as:

$j_{R}=\sigma \cdot E_{0} \cdot[1-(1-2 p) \cdot \cos (\omega \cdot \tau)]$,

and the imaginary current density component of the formulas (7) and (8) as:

$j_{U}=\sigma \cdot E_{0} \cdot(1-2 p) \cdot \sin (\omega \cdot \tau)-\varepsilon_{r}(\omega) \cdot \varepsilon_{0} \cdot \omega \cdot E_{0}$.

The phase angle can be described as:

$$
\begin{aligned}
\varphi(\omega) & =\arctan \frac{j_{U}(\omega)}{j_{R}(\omega)} \\
& =\arctan \frac{\left[\sigma \cdot(1-2 p) \cdot \sin (\omega \cdot \tau)-\frac{\varepsilon_{r}(\omega) \cdot \varepsilon_{0} \cdot(\omega \cdot \tau)}{\tau}\right]}{\sigma[1-(1-2 p) \cdot \cos (\omega \cdot \tau)]}
\end{aligned}
$$

As can be seen in Fig. 1, an electron hop from one well to another results in the formation of an electric dipole. It has been shown in Ref. [35] that in the material, where the hopping charge transfer mechanism occurs, an increase of thermally activated polarization takes place. In this case, static permittivity can be described by the following formula [32]:

$\varepsilon_{r}=1+\frac{N \cdot P(T) \cdot \tau \cdot e^{2} \cdot r^{2}}{\varepsilon_{0} \cdot k \cdot T}$

where: $N$ is the potential well concentration.

It follows from the analysis presented in Ref. [39] that the formula (13) is a generalized Debye formula for permanent dipoles, whose concentration is a constant value for a given material. Assuming that:

$N \cdot P(T) \cdot \tau=N^{ \pm}=$const

where: $N^{ \pm}$- dipole concentration; the Debye formula for static relative permittivity can be obtained at an accuracy of the numerical factor 3 in the denominator, given e.g. in Ref. [38]:

$\varepsilon_{S}=1+\frac{N^{ \pm} \cdot e^{2} \cdot r^{2}}{3 \cdot \varepsilon_{0} \cdot k \cdot T}$

It is well known [38] that, along with an increase of the angular frequency $\omega$, the relative permittivity decreases from the static permittivity value to the value characteristic for high frequency. This process is described by the Debye formula with the use of the relaxation time $\tau$ (see: the example [38]): 
$\varepsilon_{r}(\omega)=\varepsilon_{\infty}+\frac{\varepsilon_{S}-\varepsilon_{\infty}}{1+\omega^{2} \tau^{2}}$

where: $\varepsilon_{S}$ - relative static permittivity, $\varepsilon_{\infty}$ - relative highfrequency permittivity.

By substituting the static permittivity value of (13) to the formula (16) and taking into account that $r=N^{-13}$, a formula for the permittivity vs. frequency dependence can be obtained for the hopping charge exchange between potential wells in a nanocomposite:

$$
\begin{aligned}
\varepsilon_{r}(\omega) & =\varepsilon_{\infty}+\frac{\left(1+\frac{N \cdot P(T) \cdot \tau \cdot e^{2} \cdot r^{2}}{\varepsilon_{0} \cdot k \cdot T}\right)-\varepsilon_{\infty}}{1+\omega^{2} \tau^{2}} \\
& =\left(1+\chi_{\infty}\right)+\frac{\left(\frac{N \cdot P(T) \cdot \tau \cdot e^{2} \cdot r^{2}}{\varepsilon_{0} \cdot k \cdot T}\right)-\chi_{\infty}}{1+\omega^{2} \cdot \tau^{2}} \\
& =\left(1+\chi_{\infty}\right)+\frac{\chi_{r 0}-\chi_{\infty}}{1+\omega^{2} \cdot \tau^{2}}
\end{aligned}
$$

where: $\chi_{\infty}$ - dielectric susceptibility at high frequencies, $\chi_{r 0}=\left(\frac{N^{1 / 3} \cdot P(T) \cdot \tau \cdot e^{2}}{\varepsilon_{0} \cdot k \cdot T}\right)-$ static dielectric susceptibility.

The imaginary component of the current density is:

$j_{U}=\sigma \cdot E_{0} \cdot(1-2 p) \cdot \sin (\omega \cdot \tau)-\left(\varepsilon_{\infty}+\frac{\varepsilon_{S}-\varepsilon_{\infty}}{1+\omega^{2} \tau^{2}}\right) \cdot \varepsilon_{0} \cdot E_{0} \cdot \frac{\omega \cdot \tau}{\tau}$

And the capacitive component of the current density:

$j_{C}=\left(\varepsilon_{\infty}+\frac{\varepsilon_{S}-\varepsilon_{\infty}}{1+\omega^{2} \tau^{2}}\right) \cdot \varepsilon_{0} \cdot E_{0} \cdot \frac{\omega \cdot \tau}{\tau}$

reaches its maximum value at the frequency of $\omega \tau=1$. It means that to analyze the phase angle vs. frequency dependence described by the formula (12), the case of $\varphi=0$ should be taken as a reference point, which means that the current density in the numerator of the formula is of value the zero (12):

$j_{U}=\sigma \cdot(1-2 p) \cdot \sin (\omega \cdot \tau=1)-\frac{\varepsilon_{S}+\varepsilon_{\infty}}{2 \cdot \tau} \cdot \varepsilon_{0}=0$

It follows from the formula (20) that the condition for the voltage resonance $\varphi_{R}=0$ to occur at the frequency $\omega_{R}=1 / \tau$ can be written as follows:

$\sigma_{R 0}=\frac{\left(\varepsilon_{S}+\varepsilon_{\infty}\right) \cdot \varepsilon_{0}}{2 \cdot \tau \cdot \sin (\omega \cdot \tau=1)}$

It seems hardly probable to encounter the conductivity value given by the formula (21). As a rule, conductivity in nanocomposites is lower or higher than the value that results from this formula (21). An analysis of the formula (12) has shown that, in the ultra-low frequency area, a nanocomposite exhibits resistive type of conduction and the phase angle is $\varphi_{\text {ulf }}=0$. In the area of higher frequencies, the phase angle value is a function of conductivity, static permittivity, dipole lifetime and the measuring frequency. In order to illustrate those dependences, a computer simulation has been performed using the formulas (12) and (18). For the calculation purposes, the following parameter values of the formulas (12) and (18) have been applied: $\varepsilon_{S}=1000, \varepsilon_{\infty}=10, \tau=10^{-6} \mathrm{~s}, p=10^{-3}$ and the conductivity values have been varied within the range of $10^{-4} \leq \sigma \leq 10^{2} \mathrm{~S} \mathrm{~m}^{-1}$. The computer simulation results are presented in Fig. 2, which indicate the following:

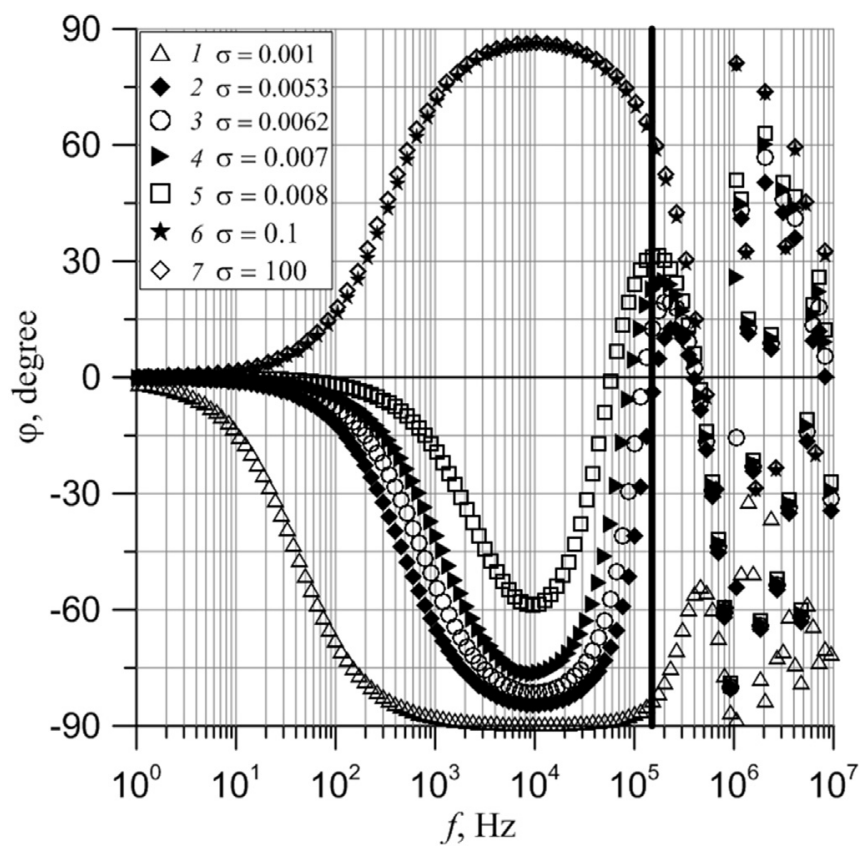

Fig. 2. Computer simulation of frequency dependencies on phase angle for conductivity values from range of $0.001 \mathrm{~S} / \mathrm{m}$ to $100 \mathrm{~S} / \mathrm{m}$.

- For the conductivity value $\sigma_{R 0}$ approaching the one given by the formula (21), negative phase angle values occur in the curves $\varphi(f)$ in the low frequency area. Once the dependence has passed through its minima, the $\varphi(f)$ values increase and they pass through the zero point, which corresponds to the voltage resonance phenomenon in conventional serial RLC circuits. Next, the $\varphi(f)$ reaches its maximum, whose location at the first approximation corresponds to the frequency of $f=1 / 2 \pi \tau$.

- For the conductivity values of $\sigma \gg \sigma_{\mathrm{R} 0}$, in the areas of low and medium frequency, positive phase angle values occur. When the maximum value has been passed at the same frequencies then, as in the case of minima of the previous case, the $\varphi(f)$ values decrease along with further frequency increase.

- For conductivity values that are low as compared to the $\sigma_{R 0}$ value, in the low frequency area, the phase angle value decreases from zero to $-90^{\circ}$ and its value stabilizes at that level.

- Oscillations that can be observed for frequencies exceeding $10^{5} \mathrm{~Hz}$, are not related to the phenomena that occur in a nanocomposite under AC flow. They result from oscillations of the function $\sin (\omega \tau)$ that is an element of the formula for the current density imaginary component (18). Taking into account the random distribution of distances between potential wells in a nanocomposite, the time of the electron return hop (dipole lifetime $\tau$ ) can assume varied values for various pairs of adjacent wells, which entails the occurrence of the time $\tau$ probability distribution and consequently the oscillation fitting for the value of $\omega>1 / \tau$. Thus, curves presented in Fig. 2 reflect the character of the dependence $\varphi(f)$ only for the values lower than $f=1 /(2 \pi \tau)$ that is represented as a vertical line.

\section{Analysis of the computer simulation results}

In Fig. 2, the curve 3 represents the dependence $\varphi(f)$ for the conductivity value $\sigma_{\mathrm{R} 0}$ described by the formula (21). As can be seen in Fig. 2, in the low frequency area, the $\varphi$ value changes from 0 to approx. $-90^{\circ}$ along with the increasing frequency; reaching its 
minimum for the frequency of approx. $10^{4} \mathrm{~Hz}$. Further frequency increase is accompanied with the phase angle $\varphi$ increase up to $\varphi=0$ at the frequency of about $10^{5} \mathrm{~Hz}$, which corresponds to voltage resonance occurrence. Along with the further frequency growth, the phase angle $\varphi$ passes to the area of positive values. For the $\sigma$ value, approaching $\sigma_{\mathrm{R} 0}$, the characteristics $\varphi(f)$ behave similarly (curves $2,4,5$ ). For values much lower than $\sigma_{R 0}$, the $\varphi$ value decreases more rapidly down to approx. $-90^{\circ}$ (curve 1 ) but still remains negative. When the conductivity values significantly exceed the $\sigma_{\mathrm{R} 0}$ value $\varphi(f)$ (curves 6,7 ) in low frequency area, the phase angle enters the area of positive values, reaching its maximum at the frequency of approx. $10^{4} \mathrm{~Hz}$. Having passed the maximum point, the $\varphi$ value gradually decreases. As can be seen in Fig. 2, having crossed the vertical line that indicates transition to the area, where the simulation yields results encumbered with high uncertainty, curves for $\sigma \gg \sigma_{\mathrm{R} 0}$ pass through zero. This result is uncertain and its positive or negative verification requires more complex simulations considering the distribution of the dipole lifetime $\tau$ probability density.

Fig. 3 presents frequency dependence of capacity measured in a parallel equivalent circuit for the case of the voltage resonance occurrence. This dependence has been calculated based on the above-presented model for the conductivity value of $\sigma_{\mathrm{R} 0}$ that is characterized by the voltage resonance occurrence. As shown in Fig. 3, at the frequency, when the voltage resonance occurs, a distinct capacity minimum occurs.

\section{Experimental}

The tested nanocomposites have been produced by ion-beam sputtering in the argon and oxygen atmosphere. Targets that consist of the metal alloy FeCoZr and dielectric PZT strips have been sputtered. As mentioned above, PZT is an acronym for the first letters of the most important components of the matrix $\left(\mathrm{Pb}_{81} \mathrm{Sr}_{4}\left(\mathrm{Na}_{50} \mathrm{Bi}_{50}\right)_{15}\left(\mathrm{Zr}_{57,5} \mathrm{Ti}_{42,5}\right)\right) \mathrm{O}_{3}$. The process gas partial pressure was $p_{\mathrm{Ar}}=7.4 \times 10^{-2} \mathrm{~Pa}$ and the oxygen pressure $p_{\mathrm{O} 2}=2 \times 10^{-3} \mathrm{~Pa}$.

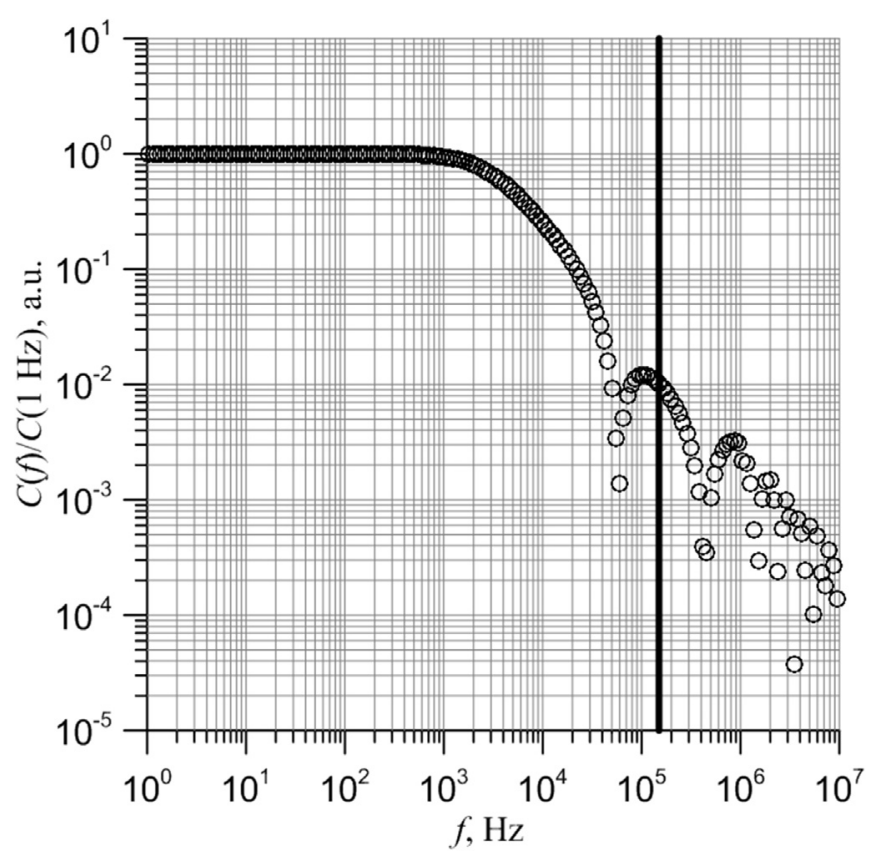

Fig. 3. Computer simulation of frequency dependence on relative capacitive for conductivity value $\sigma=0.0062 \mathrm{~S} / \mathrm{m}$.
Measurements of electrical parameters have been performed on a test stand made in the Department of Electrical Devices and High Voltage Technologies of the Lublin University of Technology. Measurements of AC parameters (such as conductivity $\sigma$, phase angle $\varphi$, capacity $C_{\mathrm{p}}$ ) of thin (about $1-2 \mu \mathrm{m}$ ) nanocomposite films $(\mathrm{FeCoZr})_{x}(\mathrm{PZT})_{(100-x)}$ have been performed using an alternating current in the frequency range of $50 \mathrm{~Hz}-1 \mathrm{MHz}$ for measuring temperatures ranging from $77 \mathrm{~K}$ to $373 \mathrm{~K}$. In the test cycle the samples have been annealed for $15 \mathrm{~min}$ in a tubular furnace, in the atmospheric air in the temperature ranging from $397 \mathrm{~K}$ to $873 \mathrm{~K}$ with a temperature change step of $25 \mathrm{~K}$. A detailed description of the measurement stand is presented in Ref. [40].

\section{Comparative analysis of the experimental data and the computer simulation results}

Fig. 4 presents selected results of the phase angle measurements performed for a nanocomposite $(\mathrm{FeCoZr})_{x}(\mathrm{PZT})_{(100-x)}$ for $x=55.9$ at.\%, annealed in a tubular furnace at temperatures of $498 \mathrm{~K}$ and $523 \mathrm{~K}$. The applied measuring temperature has been of $193 \mathrm{~K}$. As can be seen in Fig. 4, phase angle curves resemble those obtained by the computer simulation for the conductivity value approaching $\sigma_{\mathrm{R} 0}$ (curves $2-5$ in Fig. 2). It can also be seen in that figure that the increase of annealing temperature from $498 \mathrm{~K}$ to $523 \mathrm{~K}$ causes the phase angle to decrease down to its minimum value, which occurs at a frequency of about $10^{4} \mathrm{~Hz}$. It follows from Fig. 2 that the upward shift of the minimal phase angle value occurs due to the $\sigma_{\mathrm{R} 0}$ value increase (equation (21)). Fig. 5 presents frequency dependences of conductivity for a nanocomposite $(\mathrm{FeCoZr})_{x}(\mathrm{PZT})_{(100-x)}$ of $x=55.9$ at.\% annealed in a tubular furnace at temperatures $498 \mathrm{~K}$ and $523 \mathrm{~K}$. The applied measuring temperature was $193 \mathrm{~K}$. As can be seen in this figure, conductivity increase occurs in the annealing process. The conductivity is a component of the formula for $\sigma_{\mathrm{RO}}$. This means that the phase angle value decrease in the minimum shown in Fig. 4, is consistent with the model presented in this paper. As can be seen in Fig. 6, also the experimental curves of the capacity $C_{p}$ vs. frequency dependence

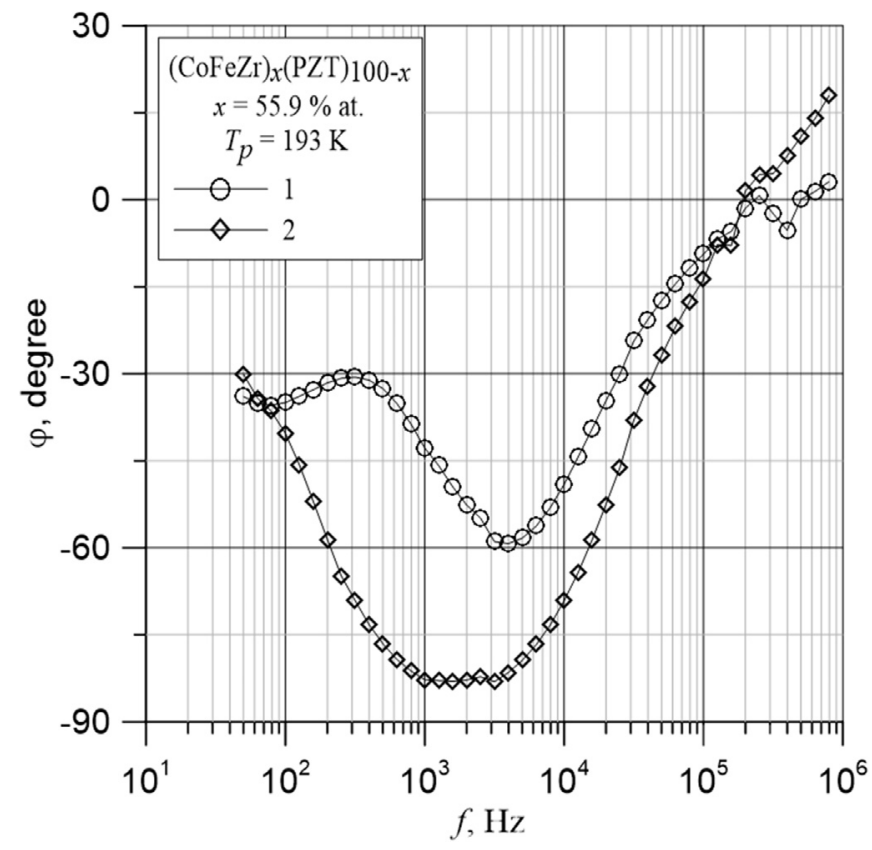

Fig. 4. Frequency dependence on phase shift $\varphi$ of nancomposite $(\mathrm{FeCoZr})_{x}(\mathrm{PZT})_{(100-x)}$ with metallic phase content $x=55.9$ at.\% measured at measurement temperature $T_{\mathrm{p}}=193 \mathrm{~K}$ after annealing in temperature $T_{\mathrm{a}}: 1-498 \mathrm{~K}$ and $2-523 \mathrm{~K}$. 


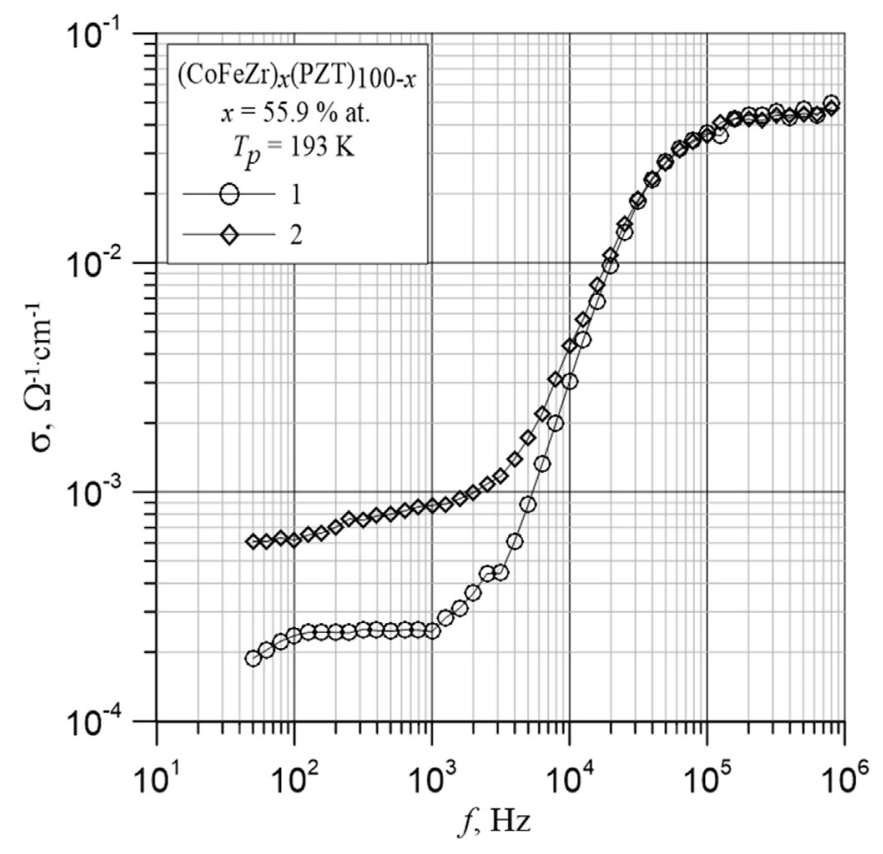

Fig. 5. Frequency dependence on conductivity $\sigma$ of nancomposite $(\mathrm{FeCoZr})_{x}(\mathrm{PZT})_{(100-x)}$ with metallic phase content $x=55.9$ at.\% measured at measurement temperature $T_{\mathrm{p}}=193 \mathrm{~K}$ after annealing in temperature $T_{\mathrm{a}}: 1-498 \mathrm{~K}$ and $2-523 \mathrm{~K}$.

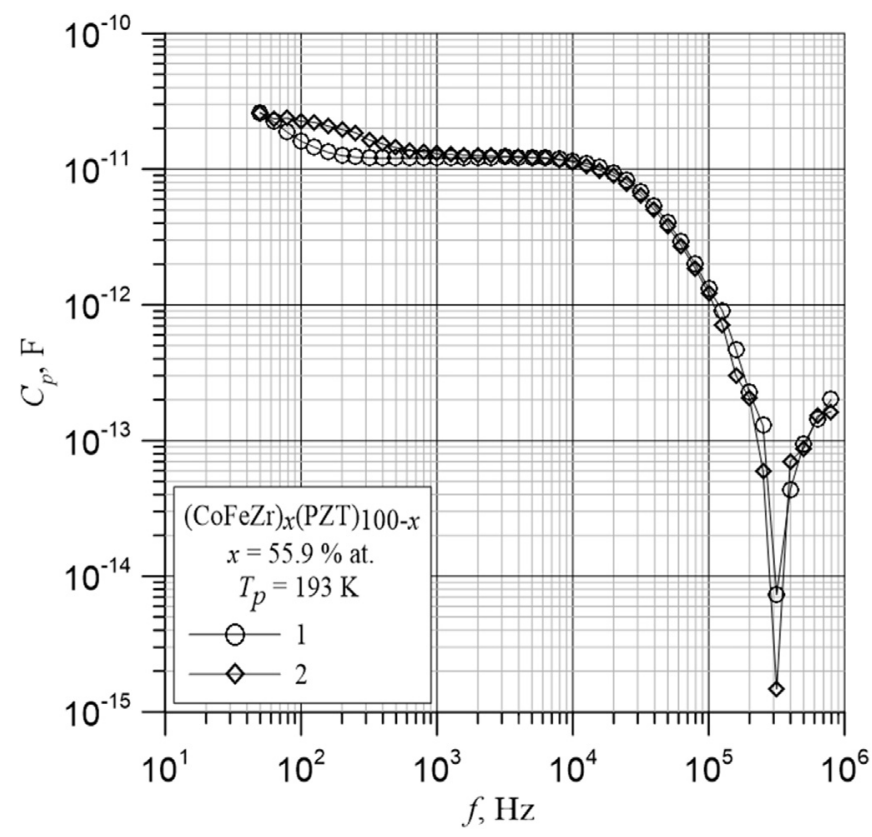

Fig. 6. Frequency dependence on capacitive $C_{\mathrm{p}}$ of nancomposite $(\mathrm{FeCoZr})_{x}(\mathrm{PZT})_{\left(100_{-x}\right)}$ metallic phase content $x=55.9$ at.\% measured at measurement temperature $T_{\mathrm{p}}=193 \mathrm{~K}$ after annealing in temperature $T_{\mathrm{a}}: 1-498 \mathrm{~K}$ and $2-523 \mathrm{~K}$.

resemble those obtained by the computer simulation shown in Fig. 3.

\section{Conclusions}

The paper presents a model of the hopping charge exchange for alternating and direct current. The model assumes that the tested material contains neutral potential wells that are located close to each other. The wells are metallic-phase nanoparticles separated by a dielectric matrix. Another assumption is that the exciting electric field is weak and does not affect the probability of electron hops from one neutral potential well to another; its only effect is some asymmetry of hops. Having hopped from one potential well to another, the electron stays there for a time period referred to as the dipole lifetime $\tau$ and next it can hop to the third well in a direction determined by the external electric field at the probability $p$ or it can return to first well at the probability $(1-p)$. Those phenomena are accompanied by the formation of three components of the hopping conductivity, two of them being phase-shifted relative to the exciting electric field.

Additionally, the model assumes that displacement current flows in the dielectric matrix. The current is related to the polarization in the metal-dielectric nanocomposites and to the formation of electric dipoles resulting from electron hops.

Based on equations developed with the model, computer simulation has been performed for frequency dependences of the phase angle and capacity within a wide range of possible changes in the nanocomposite conductivity.

An analysis of the simulation results has shown that in the area of ultra-low frequency the phase angle assumes the zero value.

For high conductivity values, the phase angle increases along with the frequency increase and reaches its maximum value of about $+90^{\circ}$, which is followed by its decrease (inductive type of conduction).

For low conductivity values, as the frequency increases, the phase angle value decreases and then stabilizes at the level of about $-90^{\circ}$ (capacitive type of conduction).

For the conductivity values that are in between the abovediscussed areas, the phase angle decreases reaching its minimum and then it begins to increase, passes through zero and reaches positive values. Its passing through the zero point corresponds to the voltage resonance phenomena in conventional RLC circuits. The resonance frequency is represented by a distinct and strong minimum in the capacity vs. frequency characteristic.

Experimental verification of the model has shown that, for a nanocomposite $(\mathrm{FeCoZr})_{x}(\mathrm{PZT})_{(100-x)}$ with the metallic phase content close to the percolation threshold ( $x=55.9$ at.\%), the phase angle vs. frequency dependences resemble the ones obtained by the computer simulation for intermediate conductivity values, where the phase angle reaches its minimum, then increases, passes through zero and reaches positive values. The experimentally obtained frequency dependences of capacity also resemble those of the computer simulation and exhibit strong minimum at the voltage resonance frequency, when the phase angle reaches zero.

\section{Acknowledgments}

The investigations have been carried out as the research project No IP2012 026572 within the framework of the Iuventus Plus program of the Polish Ministry of Science and Higher Education in the years of 2013-2015. Dr. T.N. Koltunowicz is a team member for the project: "Qualifications for the labour market - employer friendly university", cofinanced by European Union from European Social Fund.

\section{References}

[1] Imry Y, editor. Introduction to mesoscopic physics. Oxford: University Press; 2002.

[2] Żukowski P, Kołtunowicz T, Partyka J, Fedotova YuA, Larkin AV. Hopping conductivity of metal-dielectric nanocomposites produced by means of magnetron sputtering with the application of oxygen and argon ions. Vacuum 2009;83:S280-3.

[3] Kołtunowicz TN, Zhukowski P, Fedotova VV, Saad AM, Fedotov AK. Hopping conductance in nanocomposites $\left(\mathrm{Fe}_{0.45} \mathrm{Co}_{0.45} \mathrm{Zr}_{0.10}\right)_{\mathrm{x}}\left(\mathrm{Al}_{2} \mathrm{O}_{3}\right)_{1-\mathrm{x}}$ manufactured 
by ion-beam sputtering of complex target in $\mathrm{Ar}+\mathrm{O}_{2}$ ambient. Acta Phys Pol A 2011;120:39-42.

[4] Svito I, Fedotov AK, Koltunowicz TN, Zhukowski P, Kalinin Y, Sitnikov A, et al. Hopping of electron transport in granular $\mathrm{Cu}_{\mathrm{x}}\left(\mathrm{SiO}_{2}\right)_{1-\mathrm{x}}$ nanocomposite films deposited by ion-beam sputtering. J Alloys Compd 2014 615:S371-4.

[5] Koltunowicz TN, Zukowski P, Milosavljević M, Saad AM, Kasiuk JV, Fedotova JA, et al. AC/DC conductance in granular nanocomposite films $\left(\mathrm{Fe}_{45} \mathrm{CO}_{45} \mathrm{Zr}_{10}\right)_{\mathrm{x}}\left(\mathrm{CaF}_{2}\right)_{100-x}$. J Alloys Compd 2014:586:S353-6.

[6] Kołtunowicz TN, Fedotova JA, Zhukowski P, Saad A, Fedotov A, Kasiuk JV, et al. Negative capacitance in (FeCoZr)-(PZT) nanocomposite films. J Phys D Appl Phys 2013;46:125304.

[7] Young M. The techincal writers handbook. Mill Valley, CA: University Science; 1989.

[8] Duncombe JU. Infrared navigation - part I: an assessment of feasibility (Periodical style). IEEE Trans Electron Devices 1959;11:34-9.

[9] Chen S, Mulgrew B, Grant PM. A clustering technique for digital communications channel equalization using radial basis function networks. IEEE Trans Neural Netw 1993;4:570-90.

[10] Lucky RW. Automatic equalization for digital communication. Bell Syst Tech ] 1965;44:547-88.

[11] Bingulac SP. In: On the compatibility of adaptive controllers (published conference proceedings style). Proc. 4th annu. allerton conf. circuits and systems theory, New York; 1994. p. 8-16.

[12] Faulhaber GR. Design of service systems with priority reservation. IEEE Int Conf Commun 1995:3-8.

[13] Gubin SP, Koksharov YuA, Khomutov GB, Yurkov GYu. Magnetic nanoparticles: preparation, structure and properties. Russ Chem Rev 2005;74: 489-520.

[14] Mazaleyrat F, Varga LK. Ferromagnetic nanocomposites. J Magn Magn Mater 2000;215-216:253-9.

[15] McHenry ME, Willard MA, Laughlin DE. Amorphous and nanocrystalline materials for applications as soft magnets. Prog Mater Sci 1999;44 $291-433$.

[16] Pogrebnjak D. Structure and properties of nanostructured (Ti-Zr-Hf-V-Nb)N coatings. J Nanomater 2013:780125.

[17] Pogrebnjak AD, Il'jashenko M, Kul'ment'eva OP, Kshnjakin VS, Kobzev AP, Tyurin YN, et al. Structure and properties of $\mathrm{Al}_{2} \mathrm{O}_{3}$ and $\mathrm{Al}_{2} \mathrm{O}_{3}+\mathrm{Cr}_{2} \mathrm{O}_{3}$ coatings deposited to steel $3(0.3 \mathrm{wt} \% \mathrm{C})$ substrate using pulsed detonation technology. Vacuum 2001:62:21-6.

[18] Noli F, Misaelides P, Hatzidimitriou A, Pavlidou E, Pogrebnjak AD. Investigation of the characteristics and corrosion resistance of $\mathrm{Al}_{2} \mathrm{O}_{3} / \mathrm{TiN}$ coatings. Appl Surf Sci 2006;252:8043-9.

[19] Pakhomov AB, Yan X. Resistivity and Hall resistivity in percolating (NiFe)- $\mathrm{SiO}_{2}$ films. Solid State Commun 1996;99:139-42.

[20] Aronzon BA, Kovalev DYu, Lagar'kov AN, Meilikhov EZ, Ryl'kov VV, Sedova MA, et al. Anomalous Hall effect in granular $\mathrm{Fe} / \mathrm{SiO}_{2}$ films in the tunneling-conduction regime. J Exp Theor Phys Lett 1999;70(2):90-6.

[21] Aronzon BA, Granovskil AB, Davydov AB, Dokukin ME, Kalinin YuE, Nikolaev SN, et al. Planar Hall effect and anisotropic magnetoresistance in layered structures $\mathrm{Co}_{0.45} \mathrm{Fe}_{0.45} \mathrm{Zr}_{0.1} / \alpha$-Si with percolation conduction. J Exp Theor Phys 2006;103(1):110-8.

[22] Mott NF, Davis EA. Electron process in non-crystalline materials. Oxford: Claredon Press; 1979.

[23] Zukowski P, Koltunowicz T, Partyka J, Wegierek P, Komarov FF, Mironov AM, et al. Dielectric properties and model of hopping conductivity of GaAs irradiated by $\mathrm{H}^{+}$ions. Vacuum 2007;81(10):1137-40.
[24] Żukowski P, Węgierek P, Billewicz P, Kołtunowicz T, Komarov FF. Jump mechanism of electric transfer in gallium arsenide exposed on polyenergy implantation of $\mathrm{H}^{+}$ions. Acta Phys Pol A 2011;120(1):125-8.

[25] Żukowski P, Kołtunowicz T, Partyka J, Węgierek P, Kolasik M, Larkin AV, et al. A model of hopping recharging and its verification for nanostructures formed by the ion techniques. Przeglad Elektrotechniczny 2008;84(3):247-9 [In Polish].

[26] Kasyuk YuV, Fedotova JA, Marszalek M, Karczmarska A, Mitura-Nowak M, Kalinin YuE, et al. Effect of oxygen pressure on phase composition and magnetic structure of $\mathrm{FeCoZr}-\mathrm{Pb}(\mathrm{ZrTi}) \mathrm{O}_{3}$ nanocomposites. Solid State Phys 2012;54(1):178-84.

[27] Koltunowicz TN, Zukowski P, Boiko O, Saad A, Fedotova JA, Fedotov AK, et al. AC hopping conductance in nanocomposite films with ferromagnetic alloy nanoparticles in ferroelectric matrix. J Electron Mater 2015. http://dx.doi.org/ 10.1007/s11664-015-3685-9.

[28] Fedotova JA. Effect of matrix onto oxidation of metallic nanoparticles in metal-insulator nanocomposite films. Acta Phys Pol A 2014;125(6):1418-20.

[29] Koltunowicz TN. Inductive type properties of FeCoZr-CaF2 and FeCoZr-PZT nanocomposites. J Mater Sci - Mater Electron 2015 [In press].

[30] Kołtunowicz TN, Zhukowski P, Fedotov AK, Larkin AV, Patryn A Andriyevskyy B, et al. Influence of matrix type on negative capacitance effect in nanogranular composite films FeCoZr-Insulator. Elektronika ir Elektrotechnika Electron Electr Eng 2013;19(4):37-40.

[31] Zhukowski P, Kołtunowicz TN, Wegierek P, Fedotova JA, Fedotov AK, Larkin AV. Formation of noncoil-like inductance in nanocomposites $\left(\mathrm{Fe}_{0.45} \mathrm{Co}_{0.45} \mathrm{Zr}_{0.10}\right)_{x}\left(\mathrm{Al}_{2} \mathrm{O}_{3}\right)_{1-\mathrm{x}}$ manufactured by ion-beam sputtering of complex targets in $\mathrm{Ar}+\mathrm{O}_{2}$ atmosphere. Acta Phys Pol A 2011;120(1):43-5.

[32] Koltunowicz TN, Zhukowski P, Bondariev V, Saad A, Fedotova JA, Fedotov AK, et al. Enhancement of negative capacitance effect in $(\mathrm{FeCOZr})_{\mathrm{x}}\left(\mathrm{CaF}_{2}\right)_{(100-x)}$ nanocomposite films deposited by ion beam sputtering in argon and oxygen atmosphere. J Alloys Compd 2014;615:S361-5.

[33] Kołtunowicz TN, Zhukowski P, Bondariev V, Fedotova JA, Fedotov AK. Annealing of $(\mathrm{CoFeZr})_{\mathrm{x}}\left(\mathrm{CaF}_{2}\right)_{(100-\mathrm{x})}$ nanocomposites produced by the ionbeam sputtering in the $\mathrm{Ar}$ and $\mathrm{O}_{2}$ ambient. Acta Phys Pol A 2013:123:932-4.

[34] Svito I, Fedotova JA, Milosavljević M, Zhukowski P, Koltunowicz TN, Saad A, et al. Influence of sputtering atmosphere on hopping conductance in granular nanocomposite $(\mathrm{FeCoZr})_{\times}\left(\mathrm{Al}_{2} \mathrm{O}_{3}\right)_{1}$ x films. J Alloys Compd 2014:615:S344-7.

[35] Kołtunowicz TN, Zhukowski P, Fedotova VV, Saad AM, Larkin AV, Fedotov AK. The features of real part of admittance in the nanocomposites $\left(\mathrm{Fe}_{45} \mathrm{Co}_{45} \mathrm{Zr}_{10}\right)_{x}\left(\mathrm{Al}_{2} \mathrm{O}_{3}\right)_{(100-x)}$ manufactured by Ion-Beam Sputtering Technique with Ar ions acta. Phys Pol A 2011;120:35-8.

[36] Larkin AV, Fedotov AK, Fedotova JA, Koltunowicz TN, Zhukowski P. Temperature and frequency dependences of impedance real part in the FeCoZr-doped PZT nanogranular composites. Mater Pol 2012;30(2):75-81.

[37] Shklovsky BI, Efros AL. Elecronic properties of doped semiconductors. Berlin: Springer-Verlag; 1984.

[38] Havriliak Jr S, Havriliak SJ. Dielectric and mechanical relaxation in materials. Analysis, interpretation and application to polymers. Munich, Vienna, New York: Hanser Publishers; 1997.

[39] Zukowski PW, Rodzik A, Shostak YA. Dielectric constant and ac conductivity of semi-insulating $\mathrm{Cd}_{1-\mathrm{x}} \mathrm{Mn}_{\mathrm{x}} \mathrm{Te}$ semiconductors. Semiconductors 1997;31(6): 610-4.

[40] Komarov FF, Zhukowski P, Krivosheev RM, Munoz E, Koltunowicz TN, Rodionova VN, et al. Effects of surfactant and fabrication procedure on the electrical conductivity and electromagnetic shielding of single-walled carbon nanotube films. Phys Status Solidi A - Appl Mater Sci 2015;212(2): 425-32. 